\title{
Entanglement detection beyond the CCNR criterion for infinite-dimensions
}

\author{
GUO Yu ${ }^{1,2} \&$ HOU JinChuan ${ }^{3 *}$ \\ ${ }^{1}$ College of Physics and Optoelectronics, Taiyuan University of Technology, Taiyuan 030024, China; \\ ${ }^{2}$ Department of Mathematics, Shanxi Datong University, Datong 037009, China; \\ ${ }^{3}$ College of Mathematics, Taiyuan University of Technology, Taiyuan 030024, China
}

Received September 4, 2012; accepted January 4, 2013

In terms of the relation between the state and its reduced states, we obtain two inequalities which are valid for all separable states in infinite-dimensional bipartite quantum systems. One of them provides an entanglement criterion which is strictly stronger than the computable cross-norm or realignment (CCNR) criterion.

quantum state, entanglement, computable cross-norm or realignment criterion, infinite-dimensional quantum systems

Citation: Guo Y, Hou J C. Entanglement detection beyond the CCNR criterion for infinite-dimensions. Chin Sci Bull, 2013, 58: 1250-1255, doi: 10.1007/s11434-013$5738-x$

Quantum entanglement has been subjected to intensive studies in connection with quantum information theory and quantum communication theory [1]. One basic problem for quantum entanglement is to find a proper criterion to determine whether a given state of a composite system is entangled or not [2-18]. Although considerable progress has been achieved in this field, this problem is not fully explored yet except for the case of $2 \otimes 2$ and $2 \otimes 3$ systems $[2,3,19]$.

By definition, a bipartite state $\rho$ acting on a separable complex Hilbert space $H=H_{A} \otimes H_{B}$ is called separable if it can be written as

$$
\rho=\sum_{i} p_{i} \rho_{i}^{A} \otimes \rho_{i}^{B}, \quad \sum_{i} p_{i}=1, p_{i} \geqslant 0
$$

or it is a limit of the states of the above form under the trace norm topology [20], where $\rho_{i}^{A}$ and $\rho_{i}^{B}$ are (pure) states on the subsystems associated to the Hilbert spaces $H_{A}$ and $H_{B}$, respectively. A state that is not separable is said to be entangled. Particularly, if a state can be represented in the form as in eq. (1), it is called countably separable [21]. Observing that, for finite-dimensional systems, all separable states are finitely separable. However, there do exist separable states

*Corresponding author (email: jinchuanhou@yahoo.com.cn,houjinchuan@tyut.edu.cn) which are not countably separable in infinite-dimensional systems [21]. Thus it is interesting to study the question that what kind of criteria are still valid or can be extended to infinite-dimensional systems. This question has been attacked by several authors (for example, [10-15], [19, 21, 22] and the references therein).

For finite-dimensional systems, a very elegant criterion for the separability is the so-called computable cross-norm or realignment (CCNR) criterion proposed by Rudolph in [23] and Chen and $\mathrm{Wu}$ in [24]. The CCNR criterion states that if $\rho$ is a separable state on $H_{A} \otimes H_{B}$ with $\operatorname{dim} H_{A} \otimes H_{B}<+\infty$, then the trace norm $\left\|\rho^{R}\right\|_{\operatorname{Tr}}$ of the realignment matrix $\rho^{R}$ of $\rho$ is not greater than 1. By exploring the relation between the state and its reduced states, Zhang et al. [25] investigated a criterion beyond the CCNR criterion. It is showed in [25] that, if a state acting on $H_{A} \otimes H_{B}$ with $\operatorname{dim} H_{A} \otimes H_{B}<+\infty$ is separable, then

$$
\left\|\left(\rho-\rho_{A} \otimes \rho_{B}\right)^{R}\right\|_{\operatorname{Tr}} \leqslant \sqrt{\left[1-\operatorname{Tr}\left(\rho_{A}^{2}\right)\right]\left[1-\operatorname{Tr}\left(\rho_{B}^{2}\right)\right]}
$$

and

$$
\left\|\left(\rho-\rho_{A} \otimes \rho_{B}\right)^{T_{B}}\right\|_{\operatorname{Tr}} \leqslant 2 \sqrt{\left[1-\operatorname{Tr}\left(\rho_{A}^{2}\right)\right]\left[1-\operatorname{Tr}\left(\rho_{B}^{2}\right)\right]} .
$$


Here, $C^{R}$ denotes the realignment matrix of the block matrix $C=\left[C_{i j}\right]_{N_{A} \times N_{A}}$ with $C_{i j}$ s are $N_{B} \times N_{B}$ complex matrices with $\operatorname{dim} H_{A}=N_{A}$ and $\operatorname{dim} H_{B}=N_{B} ;\|\cdot\|_{T r}$ denotes the trace norm and $C^{T_{B}}$ denotes the partial transposition of $C$ with respect to the subsystem B. The inequality (2) provides a criterion which is stronger than the CCNR criterion [25] (namely, any entangled states that detected by the CCNR criterion can be detected by the inequality (2) and there exist some entangled states that can be detected by inequality (2) while they cannot be recognized by the CCNR criterion).

Very recently, we established the realignment operation and CCNR criterion for infinite-dimensional bipartite systems [26, 27]. We generalized the realignment operation of matrices to Hilbert-Schmidt operators on infinitedimensional Hilbert space $H_{A} \otimes H_{B}$ in [26] and showed that $\left\|\rho^{R}\right\|_{T r} \leqslant 1$ whenever $\rho$ is a separable state acting on $H_{A} \otimes H_{B}$. The aim of this paper is to establish the analogous inequalities as (2) and (3) for infinite-dimensional case. As one might expect, we show that the obtained inequality criterion is stronger than the CCNR criterion proposed in [26]. Furthermore, it can detect some PPT entangled states (i.e. the entangled states with positive partial transposition) which cannot be detected by the CCNR criterion. It should be pointed out that the corresponding inequalities for infinitedimensional case can not be derived straightforwardly from that of the finite-dimensional case. The situations grow more complicated in the case of infinite-dimensional case.

In detail, this paper is organized as follows. In Section 1 we propose some properties of the reduced density operators for both finite- and infinite-dimensional bipartite systems. We show that the reduced states stand close to each other whenever the composite states are closed to each other. Then in Section 2 we propose a practical criterion based on $\rho-\rho_{A} \otimes \rho_{B}$. The obtained criterion is strictly stronger than the CCNR criterion. Section 3 is a short conclusion.

Throughout the paper, we use the bra-ket notations. $\langle\cdot \mid \cdot\rangle$ stands for the inner product in the given Hilbert spaces. The set of all (bounded linear) operators on a Hilbert space $H$ is denoted by $\mathcal{B}(H)$, the set of all trace class operators on $H$ is denoted by $\mathcal{T}(H)$ and the space consisting of all Schattern- $p$ class operators on $H$ is denoted by $C_{p}(H) . A \in \mathcal{B}(H)$ is selfadjoint if $A^{\dagger}=A$ ( $A^{\dagger}$ stands for the adjoint operator of $A$ ); $A$ is said to be positive, denoted by $A \geqslant 0$, if $A^{\dagger}=A$ and $\langle\psi|A| \psi\rangle \geqslant 0$ for all $|\psi\rangle \in H$. $A^{T}$ stands for the transposition of the operator $A$. By $\mathcal{S}\left(H_{A}\right), \mathcal{S}\left(H_{B}\right)$ and $\mathcal{S}\left(H_{A} \otimes H_{B}\right)$ we denote the sets of all states acting on $H_{A}, H_{B}$ and $H_{A} \otimes H_{B}$, respectively. By $\mathcal{S}_{\text {sep }}\left(H_{A} \otimes H_{B}\right)$ we denote the set of all separable states in $\mathcal{S}\left(H_{A} \otimes H_{B}\right)$. We fix in the "local state spaces" $H_{A}$ and $H_{B}$ orthonormal bases $\{|m\rangle\}_{m=1}^{N_{A}}$ and $\{|\mu\rangle\}_{\mu=1}^{N_{B}}$, respectively, where $\operatorname{dim} H_{A}=N_{A}$ and $\operatorname{dim} H_{B}=N_{B}\left(N_{A / B}\right.$ may be $\left.+\infty\right)$ (note that we use Latin indices for the subsystem $A$ and the Greek indices for the subsystem B). The partial transposition of $\rho \in \mathcal{S}\left(H_{A} \otimes H_{B}\right)$ with respect to the subsystem B (resp. A) is denoted by $\rho^{T_{B}}$ (resp. $\left.\rho^{T_{A}}\right)$, that is, $\rho^{T_{B}}=\left(I_{A} \otimes \mathbf{T}\right) \rho$ (resp. $\left.\rho^{T_{A}}=\left(\mathbf{T} \otimes I_{B}\right) \rho\right)$, where $\mathbf{T}$ is the map of taking transpose, i.e.
$\mathbf{T} C=C^{T}$, with respect to a given orthonormal basis.

\section{The reduced density operators}

To describe subsystems of a composite system, one needs the reduced density operators. It is so useful as to be virtually indispensable in the analysis of composite systems [1]. In this section, we discuss some properties about the reduced density operators.

Let $H_{A}$ and $H_{B}$ be complex Hilbert spaces with $\operatorname{dim} H_{A} \otimes$ $H_{B}=+\infty, \rho=|\psi\rangle\langle\psi| \in \mathcal{S}\left(H_{A} \otimes H_{B}\right)$ be a pure state with unit vector $|\psi\rangle=\sum_{m, \mu} d_{m \mu}|m\rangle|\mu\rangle \in H_{A} \otimes H_{B}$. It is clear that $D_{\psi}=\left(d_{m \mu}\right)$ can be regarded as an operator from $H_{B}$ into $H_{A}$ and it is a Hilbert-Schmidt class operator with the HilbertSchmidt norm $\left\|D_{\psi}\right\|_{2}=\||\psi\rangle \|$. Under the given bases, we have

$$
\begin{aligned}
\rho_{A} & =\operatorname{Tr}_{B}(\rho)=\left(I_{A} \otimes \mathbf{T r}\right) \rho \\
& =\left(I_{A} \otimes \mathbf{T r}\right)\left(\sum_{m, \mu, n, v} d_{m \mu} \overline{d_{n v}}|m\rangle\langle n|\otimes| \mu\rangle\langle v|\right) \\
& =\sum_{m, \mu, n, v} d_{m \mu} \overline{d_{n v}} \operatorname{Tr}(|\mu\rangle\langle v|)|m\rangle\langle n| \\
& =\sum_{m, n, \mu} d_{m \mu} \overline{d_{n \mu}}|m\rangle\langle n| \\
& =\sum_{m, n}\left(\sum_{\mu} d_{m \mu} \overline{d_{n \mu}}\right)|m\rangle\langle n|=D D^{\dagger} .
\end{aligned}
$$

Similarly, $\operatorname{Tr}_{A}(\rho)=\left(\operatorname{Tr} \otimes I_{B}\right) \rho=\rho_{B}=D^{\dagger} D$. For any mixed state $\rho \in \mathcal{S}\left(H_{A} \otimes H_{B}\right)$, let

$$
\rho=\sum_{i} p_{i}\left|\psi_{i}\right\rangle\left\langle\psi_{i}|, \quad| \psi_{i}\right\rangle \in H_{A} \otimes H_{B}, p_{i}>0, \sum_{i} p_{i}=1,
$$

be the spectral decomposition. Write $\left|\psi_{i}\right\rangle=\sum_{m, \mu} d_{m \mu}^{(i)}|m\rangle|\mu\rangle$ and $D_{i}=\left(d_{m \mu}^{(i)}\right)$. It turns out that

$$
\rho_{A}=\sum_{i} p_{i} D_{i} D_{i}^{\dagger}, \quad \rho_{B}=\sum_{i} p_{i} D_{i}^{\dagger} D_{i} .
$$

For the finite-dimensional case, also see [28].

If $\rho, \varrho \in \mathcal{S}\left(H_{A} \otimes H_{B}\right)$, and $\rho$ stands close to $\varrho$, then, what about the distance between $\rho_{A / B}$ and $\varrho_{A / B}$ ? The following result is an answer to this question.

Proposition 1. Let $H_{A}$ and $H_{B}$ be complex separable Hilbert spaces with $\operatorname{dim} H_{A} \otimes H_{B} \leqslant+\infty, \rho, \rho_{k} \in \mathcal{S}\left(H_{A} \otimes H_{B}\right)$, $k=1,2, \ldots$ and $\lim _{k} \rho_{k}=\rho$ in trace norm. Then

$$
\lim _{k \rightarrow \infty} \rho_{A(k)}=\rho_{A} \quad \text { and } \quad \lim _{k \rightarrow \infty} \rho_{B(k)}=\rho_{B},
$$

in trace norm, where $\rho_{A(k)}=\operatorname{Tr}_{B}\left(\rho_{k}\right)$ and $\rho_{B(k)}=\operatorname{Tr}_{A}\left(\rho_{k}\right)$.

Proof. Take orthonormal bases $\{|m\rangle\}_{m=1}^{N_{A}}$ and $\{|\mu\rangle\}_{\mu=1}^{N_{B}}$ of $H_{A}$ and $H_{B}$, respectively. With respect to theses bases, we can write $\rho_{k}$ and $\rho$ in the matrix form $\rho_{k}=\left(\sigma_{m n}^{(k)}\right)$ and $\rho=\left(\sigma_{m n}\right)$, where $\sigma_{m n}^{(k)}, \sigma_{m n} \in \mathcal{T}\left(H_{B}\right)$. Then $\rho_{A(k)}=\left(\operatorname{Tr}\left(\sigma_{m n}^{(k)}\right)\right)$ and $\rho_{A}=\left(\operatorname{Tr}\left(\sigma_{m n}\right)\right)$. Since $\rho_{k} \rightarrow \rho$ as $k \rightarrow \infty$ under the trace norm topology, we have $\sigma_{m n}^{(k)} \rightarrow \rho_{m n}$ as $k \rightarrow \infty$ under the trace norm 
topology for each $(m, n)$-entry. Hence $\operatorname{Tr}\left(\sigma_{m n}^{(k)}\right) \rightarrow \operatorname{Tr}\left(\sigma_{m n}\right)$ for any $m, n$, that is, $\rho_{A(k)}$ converges to $\rho_{A}$ entry-wise. Note that $\mathcal{T}(H)$ is the dual space of $\mathcal{B}_{0}(H)$, here $\mathcal{B}_{0}(H)$ denotes the Banach space of all compact operators on $H$. It follows that, $\rho_{A(k)}$ converges to $\rho_{A}$ under the weak star topology $\sigma\left(\mathcal{T}(H), \mathcal{B}_{0}(H)\right)$. It is known from [29] that the weak-star topology coincides with the trace norm topology on $\mathcal{S}(H)$. Therefore, we conclude that $\rho_{A(k)} \rightarrow \rho_{A}$ as $k \rightarrow \infty$ under the trace norm topology.

Similarly, one can show that $\rho_{k} \rightarrow \rho$ as $k \rightarrow \infty$ implies that $\rho_{B(k)} \rightarrow \rho_{B}$ as $k \rightarrow \infty$. $\square$

This proposition also implies that the trace operation is completely bounded under the trace norm topology on the set of all states.

\section{Detecting entanglement by inequalities induced from the CCNR criterion}

The main result of this section is the following.

Theorem 1. Let $H_{A}$ and $H_{B}$ be complex separable Hilbert spaces with $\operatorname{dim} H_{A} \otimes H_{B}=+\infty, \rho \in \mathcal{S}_{\text {sep }}\left(H_{A} \otimes H_{B}\right)$. Then

$$
\left\|\left(\rho-\rho_{A} \otimes \rho_{B}\right)^{R}\right\|_{\operatorname{Tr}} \leqslant \sqrt{\left[1-\operatorname{Tr}\left(\rho_{A}^{2}\right)\right]\left[1-\operatorname{Tr}\left(\rho_{B}^{2}\right)\right]}
$$

and

$$
\left\|\left(\rho-\rho_{A} \otimes \rho_{B}\right)^{T_{B}}\right\|_{\operatorname{Tr}} \leqslant 2 \sqrt{\left[1-\operatorname{Tr}\left(\rho_{A}^{2}\right)\right]\left[1-\operatorname{Tr}\left(\rho_{B}^{2}\right)\right]},
$$

where $\rho_{A}=\operatorname{Tr}_{B}(\rho), \rho_{B}=\operatorname{Tr}_{A}(\rho)$, and $\rho^{R}$ stands for the realignment operator of $\rho$.

There are three equivalent definitions of the realignment operator of an operator in $C_{2}\left(H_{A} \otimes H_{B}\right)$ [26], one of them is the following:

Lemma 1 (Guo et al. [26]). $\quad$ Let $H_{A}$ and $H_{B}$ be complex Hilbert spaces with $\operatorname{dim} H_{A} \otimes H_{B}=+\infty$ and let $C \in C_{2}\left(H_{A} \otimes\right.$ $H_{B}$ ) be a Hilbert-Schmidt operator with $C=\sum_{k} A_{k} \otimes B_{k}$, where $A_{k}=\sum_{m, n} a_{m n}^{(k)}|m\rangle\left\langle n\left|\in C_{2}\left(H_{A}\right), B_{k}=\sum_{\mu, v} b_{\mu \nu}^{(k)}\right| \mu\right\rangle\langle v| \in C_{2}\left(H_{B}\right)$ and the series converges in Hilbert-Schmidt norm. Then

$$
C^{R}=\sum_{k}\left|A_{k}\right\rangle\left\langle B_{k}\right|
$$

where the series converges in Hilbert-Schmidt norm, $\left|A_{k}\right\rangle=$ $\sum_{m, n} a_{m n}^{(k)}|m\rangle|n\rangle,\left|B_{k}\right\rangle=\sum_{\mu, v} b_{\mu \nu}^{(k)}|\mu\rangle|v\rangle,\left\langle B_{k}\right|$ denotes the transposition of $\left|B_{k}\right\rangle$.

In order to prove Theorem 1, some more lemmas are needed. The following lemma is well known for mathematicians and we include a proof of it here for readers' convenience.

Lemma 2. Let $H_{A}$ and $H_{B}$ be complex separable Hilbert spaces with $\operatorname{dim} H_{A} \otimes H_{B}=+\infty, A \in C_{p}\left(H_{A}\right), B \in C_{p}\left(H_{B}\right)$ and $1 \leqslant p<+\infty$. Then $A \otimes B \in C_{p}\left(H_{A} \otimes H_{B}\right)$, and further more,

$$
\|A \otimes B\|_{p}=\|A\|_{p}\|B\|_{p}
$$

Proof. Let $A=U_{1} D_{1} V_{1}$ and $B=U_{2} D_{2} V_{2}$ be the singular value decomposition of $A$ and $B$, respectively, where $D_{1}=$ $\operatorname{diag}\left(\lambda_{1}, \lambda_{2}, \cdots, \lambda_{n}, \cdots\right)$ and $D_{2}=\operatorname{diag}\left(\lambda_{1}^{\prime}, \lambda_{2}^{\prime}, \cdots, \lambda_{n}^{\prime}, \cdots\right)$ with $\lambda_{1} \geqslant \lambda_{2} \geqslant \cdots \geqslant \lambda_{n} \geqslant \cdots$ and $\lambda_{1}^{\prime} \geqslant \lambda_{2}^{\prime} \geqslant \cdots \geqslant \lambda_{n}^{\prime} \geqslant \cdots$. It follows that

$$
\|A\|_{p}=\left(\sum_{i} \lambda_{i}^{p}\right)^{\frac{1}{p}} \quad \text { and } \quad\|B\|_{p}=\left(\sum_{i} \lambda_{i}^{\prime p}\right)^{\frac{1}{p}} .
$$

Write $U_{1} \otimes U_{2}=U, D_{1} \otimes D_{2}=D$ and $V_{1} \otimes V_{2}=V$. Then we have $A \otimes B=\left(U_{1} D_{1} V_{1}\right) \otimes\left(U_{2} D_{2} V_{2}\right)=\left(U_{1} \otimes U_{2}\right)\left(D_{1} \otimes\right.$ $\left.D_{2}\right)\left(V_{1} \otimes V_{2}\right)=U D V$. Since $D$ is a diagonal operator with diagonal entries $\left\{\lambda_{i} \lambda_{j}^{\prime}\right\}$, one sees that

$$
\begin{aligned}
\|A \otimes B\|_{p} & =\left(\sum_{i, j} \lambda_{i}^{p} \lambda_{j}^{\prime p}\right)^{\frac{1}{p}}=\left[\sum_{i} \lambda_{i}^{p}\left(\sum_{j} \lambda_{j}^{\prime p}\right)\right]^{\frac{1}{p}} \\
& =\left(\sum_{i} \lambda_{i}^{p}\right)^{\frac{1}{p}}\left(\sum_{j} \lambda_{j}^{\prime p}\right)^{\frac{1}{p}}=\|A\|_{p}\|B\|_{p},
\end{aligned}
$$

as desired.

Lemma 3. Let $H_{A}$ and $H_{B}$ be complex separable Hilbert spaces with $\operatorname{dim} H_{A} \otimes H_{B}=+\infty$ and $\left\{\rho_{k}\right\}$ be a sequence in $\mathcal{S}_{\text {sep }}\left(H_{A} \otimes H_{B}\right)$. Then $\left\{\rho_{k}\right\}$ converges to $\rho$ in trace norm implies

$$
\lim _{k \rightarrow \infty} \rho_{k}^{T_{B}}=\rho^{T_{B}}
$$

in trace norm.

Proof. Since $\rho_{k}$ converges to $\rho$ in trace norm implies $\rho_{k}$ converges to $\rho$ entry-wise, thus $\rho_{k}^{T_{B}}$ converges to $\rho^{T_{B}}$ entrywise as well. And it is obvious that $\rho$ is separable, thus $\rho^{T_{B}}$ is also a state. This implies that $\lim _{k \rightarrow \infty} \rho_{k}^{T_{B}}=\rho^{T_{B}}$ with respect to the trace norm since the trace norm topology coincides with the weak-star topology on $\mathcal{S}\left(H_{A} \otimes H_{B}\right)$.

Now we are ready to give our proof of the main result.

The proof of Theorem 1. We prove the inequality (6) first. Denote by $\mathcal{S}_{s-p}$ the set of all separable pure states in $\mathcal{S}\left(H_{A} \otimes H_{B}\right)$. If $\rho$ is separable, then it admits a representation of the Bochner integral [21]

$$
\rho=\int_{\mathcal{S}_{s-p}} \varphi\left(\rho^{A} \otimes \rho^{B}\right) d \mu\left(\rho^{A} \otimes \rho^{B}\right),
$$

where $\mu$ is a Borel probability measure on $\mathcal{S}_{s-p}, \rho^{A} \otimes \rho^{B} \in$ $\mathcal{S}_{s-p}$ and $\varphi: \mathcal{S}_{s-p} \rightarrow \mathcal{S}_{s-p}$ is a measurable function. It immediately follows from proposition 1 that

$$
\rho_{A}=\int_{\mathcal{S}_{s-p}} \varphi\left(\rho^{A} \otimes \rho^{B}\right)^{A} d \mu\left(\rho^{A} \otimes \rho^{B}\right),
$$

and

$$
\rho_{B}=\int_{\mathcal{S}_{s-p}} \varphi\left(\rho^{A} \otimes \rho^{B}\right)^{B} d \mu\left(\rho^{A} \otimes \rho^{B}\right),
$$


where $\varphi\left(\rho^{A} \otimes \rho^{B}\right)^{A}=\operatorname{Tr}_{B}\left[\varphi\left(\rho^{A} \otimes \rho^{B}\right)\right], \varphi\left(\rho^{A} \otimes \rho^{B}\right)^{B}=$ $\operatorname{Tr}_{A}\left[\varphi\left(\rho^{A} \otimes \rho^{B}\right)\right]$.

Observe that $\sigma^{A} \otimes \sigma^{B} \in \mathcal{S}_{s-p}$ and

$$
\begin{aligned}
& \rho-\rho_{A} \otimes \rho_{B} \\
& =\int_{\mathcal{S}_{s-p}} \varphi\left(\rho^{A} \otimes \rho^{B}\right)^{A} \otimes \varphi\left(\rho^{A} \otimes \rho^{B}\right)^{B} d \mu\left(\rho^{A} \otimes \rho^{B}\right) \\
& -\left(\int_{\mathcal{S}_{s-p}} \varphi\left(\rho^{A} \otimes \rho^{B}\right)^{A} d \mu\left(\rho^{A} \otimes \rho^{B}\right)\right) \\
& \otimes\left(\int_{\mathcal{S}_{s-p}} \varphi\left(\rho^{A} \otimes \rho^{B}\right)^{B} d \mu\left(\rho^{A} \otimes \rho^{B}\right)\right) \\
& =\int_{\mathcal{S}_{s-p}}\left(\int_{\mathcal{S}_{s-p}} \varphi\left(\rho^{A} \otimes \rho^{B}\right)^{A} d \mu\left(\sigma^{A} \otimes \sigma^{B}\right)\right) \\
& \otimes \varphi\left(\rho^{A} \otimes \rho^{B}\right)^{B} d \mu\left(\rho^{A} \otimes \rho^{B}\right) \\
& -\left(\int_{\mathcal{S}_{s-p}} \varphi\left(\rho^{A} \otimes \rho^{B}\right)^{A} d \mu\left(\rho^{A} \otimes \rho^{B}\right)\right) \\
& \otimes\left(\int_{\mathcal{S}_{s-p}} \varphi\left(\rho^{A} \otimes \rho^{B}\right)^{B} d \mu\left(\rho^{A} \otimes \rho^{B}\right)\right) \\
& =\int_{\mathcal{S}_{s-p}} \int_{\mathcal{S}_{s-p}} \varphi\left(\rho^{A} \otimes \rho^{B}\right)^{A} \otimes \varphi\left(\rho^{A} \otimes \rho^{B}\right)^{B} \\
& \cdot d \mu\left(\rho^{A} \otimes \rho^{B}\right) d \mu\left(\sigma^{A} \otimes \sigma^{B}\right) \\
& -\int_{\mathcal{S}_{s-p}} \int_{\mathcal{S}_{s-p}} \varphi\left(\sigma^{A} \otimes \sigma^{B}\right)^{A} \\
& \otimes \varphi\left(\rho^{A} \otimes \rho^{B}\right)^{B} d \mu\left(\rho^{A} \otimes \rho^{B}\right) d \mu\left(\sigma^{A} \otimes \sigma^{B}\right) \\
& =\int_{\mathcal{S}_{s-p}} \int_{\mathcal{S}_{s-p}}\left(\varphi\left(\rho^{A} \otimes \rho^{B}\right)^{A} \otimes \varphi\left(\rho^{A} \otimes \rho^{B}\right)^{B}\right. \\
& \left.-\varphi\left(\sigma^{A} \otimes \sigma^{B}\right)^{A} \otimes \varphi\left(\rho^{A} \otimes \rho^{B}\right)^{B}\right) \\
& \cdot d \mu\left(\rho^{A} \otimes \rho^{B}\right) d \mu\left(\sigma^{A} \otimes \sigma^{B}\right) \\
& =\int_{\mathcal{S}_{s-p}} \int_{\mathcal{S}_{s-p}}\left(\varphi\left(\rho^{A} \otimes \rho^{B}\right)^{A}-\varphi\left(\sigma^{A} \otimes \sigma^{B}\right)^{A}\right) \\
& \otimes \varphi\left(\rho^{A} \otimes \rho^{B}\right)^{B} d \mu\left(\rho^{A} \otimes \rho^{B}\right) d \mu\left(\sigma^{A} \otimes \sigma^{B}\right) \\
& =\frac{1}{2} \int_{\mathcal{S}_{s-p}} \int_{\mathcal{S}_{s-p}}\left(\varphi\left(\rho^{A} \otimes \rho^{B}\right)^{A}-\varphi\left(\sigma^{A} \otimes \sigma^{B}\right)^{A}\right) \\
& \otimes\left(\varphi\left(\rho^{A} \otimes \rho^{B}\right)^{B}-\varphi\left(\sigma^{A} \otimes \sigma^{B}\right)^{B}\right) \\
& \cdot d \mu\left(\rho^{A} \otimes \rho^{B}\right) d \mu\left(\sigma^{A} \otimes \sigma^{B}\right) \text {. }
\end{aligned}
$$

Thus we arrive at

$$
\begin{aligned}
& \left(\rho-\rho_{A} \otimes \rho_{B}\right)^{R} \\
= & \frac{1}{2} \int_{\mathcal{S}_{s-p}} \int_{\mathcal{S}_{s-p}}\left[\left(\varphi\left(\rho^{A} \otimes \rho^{B}\right)^{A}-\varphi\left(\sigma^{A} \otimes \sigma^{B}\right)^{A}\right)\right. \\
& \left.\otimes\left(\varphi\left(\rho^{A} \otimes \rho^{B}\right)^{B}-\varphi\left(\sigma^{A} \otimes \sigma^{B}\right)^{B}\right)\right]^{R} \\
& \cdot d \mu\left(\rho^{A} \otimes \rho^{B}\right) d \mu\left(\sigma^{A} \otimes \sigma^{B}\right)
\end{aligned}
$$

with respect to the Hilbert-Schmidt norm since the realignment operation is continuous in the Hilbert-Schmidt norm [26]. It turns out that

$$
\left\|\left(\rho-\rho_{A} \otimes \rho_{B}\right)^{R}\right\|_{\operatorname{Tr}}
$$

$$
\begin{aligned}
\leqslant & \frac{1}{2} \int_{\mathcal{S}_{s-p}} \int_{\mathcal{S}_{s-p}} \|\left[\left(\varphi\left(\rho^{A} \otimes \rho^{B}\right)^{A}-\varphi\left(\sigma^{A} \otimes \sigma^{B}\right)^{A}\right)\right. \\
& \left.\otimes\left(\varphi\left(\rho^{A} \otimes \rho^{B}\right)^{B}-\varphi\left(\sigma^{A} \otimes \sigma^{B}\right)^{B}\right)\right]^{R} \|_{\operatorname{Tr}} \\
& \cdot d \mu\left(\rho^{A} \otimes \rho^{B}\right) d \mu\left(\sigma^{A} \otimes \sigma^{B}\right) .
\end{aligned}
$$

On the other hand, we let $\varphi\left(\sigma^{A} \otimes \sigma^{B}\right)^{A}=|x\rangle\langle x|, \varphi\left(\sigma^{A} \otimes \sigma^{B}\right)^{A}=$ $|y\rangle\left\langle y\left|, \varphi\left(\rho^{A} \otimes \rho^{B}\right)^{B}=\right| f\right\rangle\langle f|$ and $\varphi\left(\sigma^{A} \otimes \sigma^{B}\right)^{B}=|g\rangle\langle g|$, where $|x\rangle=\left(x_{1}, x_{2}, \cdots, x_{n}, \cdots\right)^{T},|y\rangle=\left(y_{1}, y_{2}, \cdots, y_{n}, \cdots\right)^{T} \in H_{A}$, $|f\rangle=\left(f_{1}, f_{2}, \cdots, f_{n}, \cdots\right)^{T}$ and $|g\rangle=\left(g_{1}, g_{2}, \cdots, g_{n}\right.$, $\cdots)^{T} \in H_{B}$. Then

$$
\begin{aligned}
& \|\left(\varphi\left(\sigma^{A} \otimes \sigma^{B}\right)^{A}-\varphi\left(\sigma^{A} \otimes \sigma^{B}\right)^{A}\right) \otimes\left(\varphi\left(\rho^{A} \otimes \rho^{B}\right)^{B}\right. \\
& \left.-\varphi\left(\sigma^{A} \otimes \sigma^{B}\right)^{B}\right)^{R} \|_{\mathrm{Tr}} \\
& =\|\left(\left|\varphi\left(\sigma^{A} \otimes \sigma^{B}\right)^{A}\right\rangle-\left|\varphi\left(\sigma^{A} \otimes \sigma^{B}\right)^{A}\right\rangle\right) \cdot\left(\left\langle\varphi\left(\rho^{A} \otimes \rho^{B}\right)^{B}\right|\right. \\
& \left.-\left\langle\varphi\left(\sigma^{A} \otimes \sigma^{B}\right)^{B}\right|\right) \|_{\mathrm{Tr}} \\
& =\left[\sum_{i, j}\left(x_{i} \overline{x_{j}}-y_{i} \overline{y_{j}}\right)\left(\overline{x_{i}} x_{j}-\overline{y_{i}} y_{j}\right)\right]^{\frac{1}{2}} \\
& \cdot\left[\sum_{i, j}\left(f_{i} \bar{f}_{j}-g_{i} \overline{g_{j}}\right)\left(\bar{f}_{i} f_{j}-\bar{g}_{i} g_{j}\right)\right]^{\frac{1}{2}} \\
& =\left[\sum_{i, j}\left(\left|x_{i} x_{j}\right|^{2}+\left|y_{i} y_{j}\right|^{2}-x_{i} \overline{x_{j}} \overline{y_{i}} y_{j}-\overline{x_{i}} x_{j} y_{i} \bar{y}_{j}\right)\right]^{\frac{1}{2}} \\
& \cdot\left[\sum_{i, j}\left(\left|f_{i} f_{j}\right|^{2}+\left|g_{i} g_{j}\right|^{2}-f_{i} \overline{f_{j}} \overline{g_{i}} g_{j}-\bar{f}_{i} f_{j} g_{i} \overline{g_{j}}\right)\right]^{\frac{1}{2}} \\
& =\left(2-\sum_{i, j}\left(x_{i} \overline{x_{j}} \overline{y_{i}} y_{j}+\overline{x_{i}} x_{j} y_{i} \overline{y_{j}}\right)\right)^{\frac{1}{2}} \\
& \cdot\left(2-\sum_{i, j}\left(f_{i} \bar{f}_{j} \bar{g}_{i} g_{j}+\bar{f}_{i} f_{j} g_{i} \bar{g}_{j}\right)\right)^{\frac{1}{2}} \\
& =2\left[\left(1-\operatorname{Tr}\left(\varphi\left(\sigma^{A} \otimes \sigma^{B}\right)^{A} \varphi\left(\sigma^{A} \otimes \sigma^{B}\right)^{A}\right)\right)\right. \\
& \left.\cdot\left(1-\operatorname{Tr}\left(\varphi\left(\rho^{A} \otimes \rho^{B}\right)^{B} \varphi\left(\sigma^{A} \otimes \sigma^{B}\right)^{B}\right)\right)\right]^{\frac{1}{2}} .
\end{aligned}
$$

So, we have

$$
\begin{aligned}
& \left\|\left(\rho-\rho_{A} \otimes \rho_{B}\right)^{R}\right\|_{\operatorname{Tr}} \\
\leqslant & \int_{\mathcal{S}_{s-p}} \int_{\mathcal{S}_{s-p}}\left[1-\operatorname{Tr}\left(\varphi\left(\sigma^{A} \otimes \sigma^{B}\right)^{A} \varphi\left(\sigma^{A} \otimes \sigma^{B}\right)^{A}\right)\right]^{\frac{1}{2}} \\
& \cdot\left[1-\operatorname{Tr}\left(\varphi\left(\rho^{A} \otimes \rho^{B}\right)^{B} \varphi\left(\sigma^{A} \otimes \sigma^{B}\right)^{B}\right)\right]^{\frac{1}{2}} \\
& \cdot d \mu\left(\rho^{A} \otimes \rho^{B}\right) d \mu\left(\sigma^{A} \otimes \sigma^{B}\right) \\
\leqslant & {\left[\int_{\mathcal{S}_{s-p}} \int_{\mathcal{S}_{s-p}} \|\left(1-\operatorname{Tr}\left(\varphi\left(\sigma^{A} \otimes \sigma^{B}\right)^{A} \varphi\left(\sigma^{A} \otimes \sigma^{B}\right)^{A}\right)\right)\right.} \\
& \left.\cdot d \mu\left(\rho^{A} \otimes \rho^{B}\right) d \mu\left(\sigma^{A} \otimes \sigma^{B}\right)\right]^{\frac{1}{2}} \\
& \cdot\left[\int_{\mathcal{S}_{s-p}} \int_{\mathcal{S}_{s-p}} \|\left(1-\operatorname{Tr}\left(\varphi\left(\sigma^{A} \otimes \sigma^{B}\right)^{B} \varphi\left(\sigma^{A} \otimes \sigma^{B}\right)^{B}\right)\right)\right. \\
& \left.\cdot d \mu\left(\rho^{A} \otimes \rho^{B}\right) d \mu\left(\sigma^{A} \otimes \sigma^{B}\right)\right]^{\frac{1}{2}} \\
= & {\left[\left(1-\operatorname{Tr}\left(\rho_{A}^{2}\right)\right)\left(1-\operatorname{Tr}\left(\rho_{B}^{2}\right)\right)\right]^{\frac{1}{2}} . }
\end{aligned}
$$

For the second inequality above the Cauchy-Schwarz inequality is used. 
Now we begin to show the inequality (7). If $\rho$ is countably separable with $\rho=\sum_{i} p_{i} \rho_{i}^{A} \otimes \rho_{i}^{B}$, then, by Lemma 2, we have

$$
\begin{aligned}
& \left\|\left(\rho-\rho_{A} \otimes \rho_{B}\right)^{T_{B}}\right\|_{\mathrm{Tr}} \\
= & \left\|\frac{1}{2} \sum_{i, j} p_{i} p_{j}\left(\rho_{i}^{A}-\rho_{j}^{A}\right) \otimes\left(\overline{\rho_{i}^{B}}-\bar{\rho}_{j}^{B}\right)\right\|_{\mathrm{Tr}} \\
\leqslant & \frac{1}{2} \sum_{i, j} p_{i} p_{j}\left\|\left(\rho_{i}^{A}-\rho_{j}^{A}\right) \otimes\left(\rho_{i}^{B}-\overline{\rho_{j}^{B}}\right)\right\|_{\operatorname{Tr}} \\
= & \frac{1}{2} \sum_{i, j} p_{i} p_{j}\left\|\rho_{i}^{A}-\rho_{j}^{A}\right\|_{\operatorname{Tr}}\left\|\rho_{i}^{B}-\rho_{j}^{B}\right\|_{\operatorname{Tr}} \\
= & \frac{1}{2} \sum_{i, j} p_{i} p_{j}\left\|\rho_{i}^{A}-\rho_{j}^{A}\right\|_{\operatorname{Tr}}\left\|\rho_{i}^{B}-\rho_{j}^{B}\right\|_{\operatorname{Tr}}
\end{aligned}
$$

since

$$
\begin{aligned}
& \rho-\rho_{A} \otimes \rho_{B} \\
= & \sum_{i} p_{i} \rho_{i}^{A} \otimes \rho_{i}^{B}-\left(\sum_{i} p_{i} \rho_{i}^{A}\right) \otimes\left(\sum_{j} p_{j} \rho_{j}^{B}\right) \\
= & \sum_{i, j}\left(p_{j} \rho_{i}^{A}\right) \otimes\left(p_{i} \rho_{i}^{B}\right)-\left(\sum_{i} p_{i} \rho_{i}^{A}\right) \otimes\left(\sum_{j} p_{j} \rho_{j}^{B}\right) \\
= & \sum_{i, j}\left[\left(p_{j} \rho_{i}^{A}\right) \otimes\left(p_{i} \rho_{i}^{B}\right)-\left(p_{i} \rho_{i}^{A}\right) \otimes\left(p_{j} \rho_{j}^{B}\right)\right] \\
= & \sum_{i, j} p_{i} p_{j}\left(\rho_{i}^{A} \otimes \rho_{i}^{B}-\rho_{i}^{A} \otimes \rho_{j}^{B}\right) \\
= & \frac{1}{2} \sum_{i, j} p_{i} p_{j}\left(\rho_{i}^{A}-\rho_{j}^{A}\right) \otimes\left(\rho_{i}^{B}-\rho_{j}^{B}\right) .
\end{aligned}
$$

Noticing that $\operatorname{rank}\left(\rho_{i}^{A}-\rho_{j}^{A}\right) \leqslant 2, \operatorname{Tr}\left(\rho_{i}^{A}-\rho_{j}^{A}\right)=0$ and $\left(\rho_{i}^{A}-\rho_{j}^{A}\right)^{\dagger}=\rho_{i}^{A}-\rho_{j}^{A}$, we can conclude that the eigenvalues of $\rho_{i}^{A}-\rho_{j}^{A}$ are $\alpha,-\alpha, \alpha \geqslant 0$, which implies that the singular values of $\rho_{i}^{A}-\rho_{j}^{A}$ are $\alpha, \alpha$. It follows from $\operatorname{Tr}\left[\left(\rho_{i}^{A}-\rho_{j}^{A}\right)^{2}\right]=2 \alpha^{2}$ that $\left\|\rho_{i}^{A}-\rho_{j}^{A}\right\|_{\operatorname{Tr}}=\sqrt{2 \operatorname{Tr}\left[\left(\rho_{i}^{A}-\rho_{j}^{A}\right)^{2}\right]}=2 \sqrt{1-\operatorname{Tr}\left(\rho_{i}^{A} \rho_{j}^{A}\right)}$. Similarly, we have $\left\|\rho_{i}^{B}-\rho_{j}^{B}\right\|_{\operatorname{Tr}}=2 \sqrt{1-\operatorname{Tr}\left(\rho_{i}^{B} \rho_{j}^{B}\right)}$. Thus, by Cauchy-Schwarz inequality, we arrive at

$$
\left\|\left(\rho-\rho_{A} \otimes \rho_{B}\right)^{T_{B}}\right\|_{\operatorname{Tr}} \leqslant 2 \sqrt{\left[1-\operatorname{Tr}\left(\rho_{A}^{2}\right)\right]\left[1-\operatorname{Tr}\left(\rho_{B}^{2}\right)\right]} .
$$

If $\rho$ is not countably separable, then there exists a sequence of countably separable states $\left\{\sigma_{n}\right\}$ such that $\lim _{n \rightarrow \infty} \sigma_{n}=\rho$ with respect to the trace norm. It follows from Proposition 1 and Lemma 3 that,

$$
\begin{aligned}
& \left\|\left(\rho-\rho_{A} \otimes \rho_{B}\right)^{T_{B}}\right\|_{\operatorname{Tr}} \\
= & \lim _{n \rightarrow \infty}\left\|\left(\sigma_{n}-\sigma_{A(n)} \otimes \sigma_{B(n)}\right)^{T_{B}}\right\|_{\operatorname{Tr}} \\
\leqslant & \lim _{n \rightarrow \infty} 2 \sqrt{\left[1-\operatorname{Tr}\left(\sigma_{A(n)}^{2}\right)\right]\left[1-\operatorname{Tr}\left(\sigma_{B(n)}^{2}\right)\right]} \\
= & \sqrt{\left[1-\operatorname{Tr}\left(\rho_{A}^{2}\right)\right]\left[1-\operatorname{Tr}\left(\rho_{B}^{2}\right)\right]},
\end{aligned}
$$

where $\sigma_{A(n)}=\operatorname{Tr}_{B}\left(\sigma_{n}\right)$ and $\sigma_{B(n)}=\operatorname{Tr}_{A}\left(\sigma_{n}\right)$.
We assert that inequality (6) can detect all states that can be recognized by the CCNR criterion. In fact, if $\left\|\rho^{R}\right\|_{\mathrm{Tr}}>1$, then $\left\|\left(\rho-\rho_{A} \otimes \rho_{B}\right)^{R}\right\|_{\mathrm{Tr}} \geqslant\left\|\rho^{R}\right\|_{\mathrm{Tr}}-\left\|\left(\rho_{A} \otimes \rho_{B}\right)^{R}\right\|_{\mathrm{Tr}}=\left\|\rho^{R}\right\|_{\mathrm{Tr}}-$ $\left.\| \rho_{A}\right\rangle\left\langle\rho_{B}\left\|_{\mathrm{Tr}}=\right\| \rho^{R}\left\|_{\mathrm{Tr}}-\right\| \rho_{A}\left\|_{2} \cdot\right\| \rho_{B}\left\|_{2}>1-\right\| \rho_{A}\left\|_{2} \cdot\right\| \rho_{B} \|_{2} \geqslant\right.$ $\sqrt{\left[1-\operatorname{Tr}\left(\rho_{A}^{2}\right)\right]\left[1-\operatorname{Tr}\left(\rho_{B}^{2}\right)\right]}$. In what follows, we will show that the inequality (6) in Theorem 1 provides a criterion that can detect some PPT entangled state $\rho$ with $\left\|\rho^{R}\right\|_{T r} \leqslant 1$.

Example. Let $H_{A}$ and $H_{B}$ be complex Hilbert spaces with orthonormal bases $\{|0\rangle,|1\rangle,|2\rangle, \ldots\}$ and $\left\{\left|0^{\prime}\right\rangle,\left|1^{\prime}\right\rangle,\left|2^{\prime}\right\rangle, \ldots\right\}$, respectively. Let $0<a<1$ and

$$
\begin{aligned}
\tilde{\rho}= & \frac{a}{8 a+1}\left(\left|00^{\prime}\right\rangle\left\langle 00^{\prime}|+| 01^{\prime}\right\rangle\left\langle 01^{\prime}|+| 02^{\prime}\right\rangle\left\langle 02^{\prime}\right|\right. \\
& +\left|00^{\prime}\right\rangle\left\langle 11^{\prime}|+| 00^{\prime}\right\rangle\left\langle 22^{\prime}|+| 11^{\prime}\right\rangle\left\langle 00^{\prime}|+| 22^{\prime}\right\rangle\left\langle 00^{\prime}\right| \\
& +\left|10^{\prime}\right\rangle\left\langle 10^{\prime}|+| 11^{\prime}\right\rangle\left\langle 11^{\prime}|+| 12^{\prime}\right\rangle\left\langle 12^{\prime}\right| \\
& \left.+\left|11^{\prime}\right\rangle\left\langle 22^{\prime}|+| 22^{\prime}\right\rangle\left\langle 11^{\prime}|+| 21^{\prime}\right\rangle\left\langle 21^{\prime}\right|\right) \\
& +\frac{1+a}{2}\left(\left|20^{\prime}\right\rangle\left\langle 20^{\prime}|+| 22^{\prime}\right\rangle\left\langle 22^{\prime}\right|\right) \\
& +\frac{\sqrt{1-a^{2}}}{2}\left(\left|20^{\prime}\right\rangle\left\langle 22^{\prime}|+| 22^{\prime}\right\rangle\left\langle 20^{\prime}\right|\right) .
\end{aligned}
$$

Write

$$
\tilde{\rho_{\epsilon}}=\epsilon \tilde{\rho}+(1-\epsilon) \frac{P_{3}}{9}, \quad P_{3}=\sum_{i, j=0}^{2}|i\rangle\left\langle i|\otimes| j^{\prime}\right\rangle\left\langle j^{\prime}\right| .
$$

If $\operatorname{dim} H_{A}=\operatorname{dim} H_{B}=3$, it is obvious that

$$
\tilde{\rho}=\hat{a}\left(\begin{array}{ccc|ccc|ccc}
a & 0 & 0 & 0 & a & 0 & 0 & 0 & a \\
0 & a & 0 & 0 & 0 & 0 & 0 & 0 & 0 \\
0 & 0 & a & 0 & 0 & 0 & 0 & 0 & 0 \\
\hline 0 & 0 & 0 & a & 0 & 0 & 0 & 0 & 0 \\
a & 0 & 0 & 0 & a & 0 & 0 & 0 & a \\
0 & 0 & 0 & 0 & 0 & a & 0 & 0 & 0 \\
\hline 0 & 0 & 0 & 0 & 0 & 0 & \frac{1}{2}(1+a) & 0 & \frac{\sqrt{1-a^{2}}}{2} \\
0 & 0 & 0 & 0 & 0 & 0 & 0 & a & 0 \\
a & 0 & 0 & 0 & a & 0 & \frac{\sqrt{1-a^{2}}}{2} & 0 & \frac{1}{2}(1+a)
\end{array}\right)
$$

(is a bound entangled state [30]) and

$$
\tilde{\rho_{\epsilon}}=\epsilon \rho+(1-\epsilon) \frac{I}{9}, \quad \hat{a}=\frac{1}{8 a+1} .
$$

It is showed in [24] that, for $3 \otimes 3$ system, $\tilde{\rho_{\epsilon}}$ is entangled when $\epsilon \geqslant 0.9955$ and $a=0.236$ applying the CCNR criterion. Using inequality (2), it is found that $\tilde{\rho_{\epsilon}}$ is still entangled when $\epsilon=0.9939$ and $a=0.232$ [25]. It is straightforward that

$\tilde{\rho_{\epsilon}}$ is entangled whenever $\epsilon \geqslant 0.9939$ and $a=0.232$.

Define

$$
\sigma=\sum_{i=3}^{+\infty} p_{i}|i\rangle\left\langle i|\otimes| i^{\prime}\right\rangle\left\langle i^{\prime}\right|, \quad p_{i} \geqslant 0, \sum_{i=3}^{+\infty} p_{i}=1 .
$$


It is clear that $\sigma$ is separable. Now we let

$$
\tilde{\rho_{\epsilon, c}}=c \tilde{\rho_{\epsilon}}+(1-c) \sigma, \quad 0 \leqslant c \leqslant 1,
$$

then $\left\|\tilde{\rho}_{\epsilon, c}{ }^{R}\right\|_{\operatorname{Tr}}=c\left\|\tilde{\rho}_{\epsilon}^{R}\right\|_{\operatorname{Tr}}+1-c$ since $\left\|\sigma^{R}\right\|_{\operatorname{Tr}}=1$ and it is evident that $\tilde{\rho}_{\epsilon, c} T_{A \backslash B} \geqslant 0$. On the other hand, one can find that $\check{\rho_{A}}=\operatorname{Tr}_{A}\left(\tilde{\rho_{\epsilon, c}}\right)=c \operatorname{Tr}_{A}\left(\tilde{\rho_{\epsilon}}\right)+(1-c) \operatorname{Tr}_{A}(\sigma)$ and $\check{\rho_{B}}=\operatorname{Tr}_{B}\left(\tilde{\rho_{\epsilon, c}}\right)=$ $c \operatorname{Tr}_{B}\left(\tilde{\rho_{\epsilon}}\right)+(1-c) \operatorname{Tr}_{B}(\sigma)$. Together with the fact that the trace operation is completely bounded, we can conclude that there exists some $0<c_{0}<1,0.9939 \leqslant \epsilon_{0}<0.9955$ and $0<\varepsilon<0.232$ such that $\tilde{\rho}_{\epsilon, c}$ violates the inequality (6) whenever $c>c_{0}, 0.9939 \leqslant \epsilon<\epsilon_{0}$ and $0.232-\varepsilon<a<0.232+\varepsilon$ while $\left\|\tilde{\rho}_{\epsilon, c}{ }^{R}\right\|_{\operatorname{Tr}} \leqslant 1$ and $\tilde{\rho_{\epsilon, c}} T_{A \backslash B} \geqslant 0$ whenever $c>c_{0}$, $0.9939 \leqslant \epsilon<\epsilon_{0}$ and $0.232-\varepsilon<a<0.232+\varepsilon$.

\section{Conclusions}

In conclusion, an entanglement criterion beyond the CCNR criterion for infinite-dimensional systems is proposed: Based on the CCNR criterion for infinite-dimensional systems, we highlighted the relation between separable states and the reduced states via realignment operation or partial transposition. It is showed that the obtained inequality can detect more entangled states than the CCNR criterion. It should be stressed here that the proof of our main result needs new tools which is very different from the finite-dimensional case.

The authors give their thanks to the referees for helpful comments to improve the paper. This work was partially supported by the National Natural Science Foundation of China (11171249, 11271217), Research Fund for the Doctoral Program of Higher Education of China (20101402110012), China Postdoctoral Science Foundation (2012M520603) and Research Start-up Fund for Doctors of Shanxi Datong University (2011-B-01).

1 Nielsen M A, Chuang I L. Quantum Computatation and Quantum Information. Cambridge: Cambridge University Press, 2000

2 Horodecki R, Horodecki P, Horodecki M, et al. Quantum entanglement. Rev Mod Phys, 2009, 81: 865-942

3 Gühne O, Tóth G. Entanglement detection. Phys Rep, 2009, 474: 1-75

4 Li M, Fei S M, Li-Jost X Q, Bell inequality, separability and entanglement distillation. Chin Sci Bull, 2011, 56: 945-954

5 Han F. Entanglement dynamics and transfer in a double JaynesCummings model. Chin Sci Bull, 2010, 55: 1758-1762

6 Salemian S, Mohammadnejad S. An error-free protocol for quantum entanglement distribution in long-distance quantum communication. Chin Sci Bull, 2011, 56: 618-625

7 Guo Z H, Cao H X, Chen Z L, et al. Operational properties and matrix representations of quantum measures. Chin Sci Bull, 2011, 56: 16711678
8 Ma X S, Ren M F, Zhao G X, et al. Effect of decoherence from a spin environment on the entanglement dynamics of two-qutrit states. Sci China Phys Mech Astron, 2011, 54: 1833-1838

9 Di Y M, Liu S P, Liu D D. Entanglement for a two-parameter class of states in a high-dimension bipartite quantum system. Sci China Phys Mech Astron, 2010, 53: 1868-1872

10 Hou J C. A characterization of positive linear maps and criteria for entangled quantum states. J Phys A: Math Theor, 2010, 43: 385201

11 Hou J C, Qi X F. Constructing entanglement witness for infinitedimensional systems. Phys Rev A, 2010, 81: 062351

12 Hou J C, Guo Y. When different entanglement witnesses detect the same entangled states. Phys Rev A, 2010, 82: 052301

13 Hou J C, Guo Y. Constructing entanglement witnesses for states in infinite-dimensional bipartite quantum systems. Int J Theor Phys, 2011, 50: $1245-1254$

14 Qi X F, Hou J C. Positive finite rank elementary operators and characterizing entanglement of states. J Phys A: Math Theor, 2011, 44: 215305

15 Qi X F, Hou J C. Characterization of optimal entanglement witnesses. Phys Rev A, 2012, 85: 022334

16 Guo Y, Hou J C. Comment on "Remarks on the structure of states of composite quantum systems and envariance" [Phys Lett A, 355(2006)180]. Phys Lett A, 2011, 375: 1160-1162

17 Guo Y, Hou J C, Wang Y C. Concurrence for infinite-dimensioanl quantum systems. arXiv: 1203.3933v1(2012)

18 Guo Y, Hou J C. Detecting quantum correlations by means of local commuatativity. arXiv: 1107.0355v3(2011)

19 Horodecki M, Horodecki P, Horodecki R. Separability of mixed states: Necessary and sufficient conditions. Phys Lett A, 1996, 223: 1-8

20 Werner R F. Quantum states with Einstein-Posolsky-Rosen correlations asmitting a hidden-variable model. Phys Rev A, 1989, 40: 4277

21 Holevo A S, Shirokov M E, Werner R F. Separability and entanglementbreaking in infinite-dimensions. Russ Math Surv, 2005, 60: 153-154

22 Wang Y Z, Hou J C, Guo Y. An entanglement criterion for states in infinite-dimensional multipartite quantum systems. Chin Sci Bull, 2012, 57: 1643-1647

23 Rudolph O. Computable cross-norm criterion for separability. Lett Math Phys, 2004, 70: 57-64

24 Chen K, Wu L A. A matrix realignment method for recognizing entanglement. Quant Inf Comput, 2003, 3: 193-202

25 Zhang C J, Zhang Y S, Zhang S, et al. Entanglement detection beyond the computable cross-norm or realignment criterion. Phys Rev A, 2008, 77: 060301(R)

26 Guo Y, Hou J C. The CCNR criterion of separability for states in infinite-dimensional quantum systems. arXiv: 1009.0116v1(2010)

27 Guo Y, Qi X F, Hou J C. Sufficient and necessary conditions of separability for bipartite pure states in infinite-dimensional systems. Chin Sci Bull, 2011, 56: 840-846

28 Aniello P, Lupo C. A class of inequalities inducing new separability criterion for bipartite quantum systems. J Phys A: Math Theor, 2008, 41: 355303

29 Zhu S, Ma Z H. Topologies on quantum states. Phys Lett A, 2010, 374 : 1336-1341

30 Horodecki P. Separability criterion and inseparable mixed states with positive partial transposition. Phys Lett A, 1997, 232: 333-339

Open Access This article is distributed under the terms of the Creative Commons Attribution License which permits any use, distribution, and reproduction in any medium, provided the original author(s) and source are credited. 